\title{
DETECCIÓN DE GENES BLA EN AISLADOS CLÍNICOS DE Escherichia coli PROVENIENTES DE PACIENTES DEL HOSPITAL NACIONAL ZACAMIL DE EL SALVADOR
}

René Santos ${ }^{1}$

Recibido 04/07/16

Aceptado $17 / 10 / 16$

\section{RESUMEN}

La producción de enzimas inactivantes tipo Betalactamasas es uno de los mecanismos más importantes de resistencia antimicrobiana reportado a nivel mundial. Las infecciones ocasionadas por microorganismos productores de Betalactamasas de espectro extendido se relacionan con mayor mortalidad. Debido a la capacidad de diseminarse a través de elementos transponibles, los genes bla se reportan ampliamente. Sin embargo, existen países sin reportes de la circulación de éstos, a pesar de su importancia clínica.

Este estudio provee las primeras evidencias de infecciones por Gram negativos intrahospitalarios asociados a genes de betalactamasas en El Salvador. Objetivo: Determinar la presencia de genes bla tipo TEM, SHV, CTX-M $\mathrm{M}_{1-2}$ y AmpC en aislados clínicos de Escherichia coli provenientes de pacientes del Hospital Nacional Zacamil. Método: Se estudiaron 30 aislados consecutivos de Escherichia coli provenientes de pacientes durante el periodo de enero a mayo del 2015. La confirmación de especie y determinación de resistencia, se realizó con Prompt ${ }^{\mathrm{TM}}$ Inoculation Sistem-D para equipo MicroScan ${ }^{\oplus}$. La producción de BLEE se determinó por método de sinergia de doble disco. Se verificó la presencia de genes bla por medio de Reacción en Cadena de las Polimerasas. Resultados: 24 muestras resultaron positivas a BLEE determinado por método de Doble Disco y 20 por método automatizado MicroScan ${ }^{\circledR}$. Siendo más eficaz el método de doble disco para la determinación fenotípica de producción de BLEE. Ciprofloxacina y ceftriazona fueron los antibióticos más utilizados como tratamiento. Conclusión: El 36.6\% de aislados resultó positivo a genes bla tipo TEM. Se obtuvo resultados negativos a los otros genes estudiados.

Palabras clave: Control de Infección, Escherichia coli, El Salvador.

\section{DETECTION OF BLA GENES IN CLINICAL ISOLATES OF Escherichia coli FROM PATIENTS FROM HOSPITAL NACIONAL ZACAMIL DE EL SALVADOR}

\section{SUMMARY}

The production of inactivating enzymes type Beta-lactamases is one of the most important mechanisms of antimicrobial resistance reported worldwide. Infections caused by microorganisms producing extended-spectrum Beta-lactamases are associated with higher mortality. Because of the ability to spread through transposable elements, bla genes are widely reported. However, there are countries with no reports of their circulation, despite their clinical importance.

This study provides the first evidence of Gram-negative in-hospital infections associated with Beta-lactamase genes in El Salvador. Objective: Determine the presence of bla genes type TEM,

1 Doctor en Medicina, Magister en Ciencias mención Microbiología. Jefe del Departamento de Microbiología de la Universidad Evangélica de El Salvador, renesantosmd@gmail.com 
SHV, CTX-M1-2 and AmpC in Escherichia coli clinical isolates patients at Hospital Nacional Zacamil. Methods: Thirty consecutive Escherichia coli isolates from patients were studied from January to May 2015. Species confirmation and resistance determination were performed with Prompt $^{\mathrm{TM}}$ Inoculation Sistem-D for MicroScan ${ }^{\circledR}$ equipment. ESBL production was determined by double-disc synergistic method. The presence of bla genes was verified by Chain Reaction of Polymerases. Results: Twenty-four samples were positive for ESBL determined by Double Disc method and 20 by MicroScan ${ }^{\star}$ automated method. The double-disc method for the phenotypic determination of ESBL production is more effective. Ciprofloxacin and ceftriazone were the most commonly used antibiotics as treatment. Conclutions: The $36.6 \%$ of isolates tested positive for bla genes type TEM. Negative results were obtained for the other genes studied.

Keywords: Escherichia coli, El Salvador, Infection Control.

\section{INTRODUCCIÓN}

Con la explosión comercial de los antibióticos a mediados del siglo pasado, propiciado por su éxito inicial en el control de las infecciones, también aparecieron los primeros reportes de microorganismos resistentes a los antibióticos que se mostraban como la panacea del siglo en el tratamiento de las infecciones bacterianas.

En algunos casos los primeros reportes de resistencia surgieron casi inmediatamente al inicio de la utilización de los nuevos antibióticos. El éxito reportado derivó, en algunos casos, en uso injustificado acompañado de la comercialización sin restricción en algunos países como El Salvador. Estas condiciones son favorables para el surgimiento y diseminación de la resistencia bacteriana.

Los medicamentos betalactámicos se encuentran entre los más utilizados para el tratamiento de infecciones provocadas por una gran variedad de microorganismos, debido a su amplio espectro de acción. Estos medicamentos están conformados en su estructura química por el anillo betalactámico, siendo los más abundantes y los más utilizados para el tratamiento de infecciones bacterianas, producidas tanto por bacterias Gram positivas como negativas.

Su amplia variedad de preparados comerciales, la disponibilidad de presentaciones de uso oral, su relativo bajo costo y su amplio espectro de acción, propician un uso frecuente no solo por médicos sino por la población en general que no ve restricciones a su compra sin receta médica. Especialmente cuando estos son de presentación oral.

Las bacterias exhiben diversos mecanismos de resistencia a estos medicamentos, siendo uno de los más frecuentes la producción de enzimas inactivantes de tipo Betalactamasa ${ }^{1}$.

Shah y Brunn-Buisson fueron los primeros en describir Betalactamasas de trasmisión plasmidial en Europa a principios de 1980, con capacidad de hidrolizar cefalosporinas de tercera generación, apenas dos años después de la introducción de estos medicamentos ${ }^{2}$. Estas enzimas se denominaron Betalactamasas de Espectro Extendido (BLEE) y, desde esa fecha, se han reportado en casi todo el mundo. Actualmente se conocen más de trescientos tipos diferentes de BLEE.

La presencia de infecciones asociadas a microorganismos multiresistentes, representa una verdadera emergencia en salud pública, ya que la mortalidad asociada así como el alto costo de atención impacta significativamente en la economía de los establecimientos que atienden estas infecciones. En el caso de infecciones por enterobactereas productoras de BLEE el costo de atención aumenta 1.7 veces más, aproximadamente ${ }^{3,4}$.

En todo el mundo se generan diariamente reportes de establecimientos que identifican microorganismos productores de BLEE, lo que les permite a los clínicos de dicho establecimiento 
comprender mejor la ecología bacteriana y diseñar protocolos adecuados para cada establecimiento ${ }^{5}$.

Además dela resistencia mostrada a cefalosporinas de tercera y cuarta generación, penicilinas de amplio espectro y aztreonam, estas bacterias productoras de BLEE también presentan genes de resistencia a otros antibióticos, fenómeno conocido como reacción cruzada ${ }^{1,2,6,7}$. De esta manera, al ejercer presión selectiva sobre estas cepas productoras de BLEE utilizando antibióticos betalactámicos, también se seleccionan cepas resistentes a otros antibióticos, que disminuye aún más las opciones terapéuticas.

A pesar de estos reportes, en El Salvador no existe ningún estudio que caracterice los microorganismos resistentes de acuerdo al tipo de BLEE que producen y menos su riesgo potencial de acuerdo a su contenido genético.

La utilización de herramientas moleculares como la reacción en cadena de las polimerasas, permitirá una mejor comprensión del fenómeno de resistencia bacteriana para cada establecimiento, proporcionando al clínico, mayores y mejores elementos de análisis para la decisión sobre el protocolo de antibióticos a utilizar, reduciendo las complicaciones asociadas a infecciones multiresistentes.

Cada vez hay menos opciones terapéuticas para microorganismos multiresistentes yúltimamente han aparecido aislados bacterianos resistentes a todos los medicamentos conocidos. Incluyendo carbapenemasas y recientemente colistín, uno de las últimas opciones terapéuticas para bacterias Gram negativas multiresistentes ${ }^{8,9,10}$.

Existe, entonces, la necesidad de reducir el impacto que la resistencia bacteriana pueda tener en la atención médica. Mejorando el control y disminuyendo la diseminación de cepas multiresistentes a través de la transferencia genética de factores de resistencia bacteriana.

Las Enterobacterias son agentes causales frecuentes de enfermedad, relacionados a infecciones diversas tanto de origen comunitario como nosocomial. Algunoscomo Escherichiacoli, presente en la microbiota normal de múltiples especies de animales, incluyendo el humano, puede encontrarse ocasionando infecciones tanto intestinales como extraintestinales, siendo el principal agente causal de ITU ${ }^{11,12,13}$. Cuando las infecciones son de origen nosocomial, la morbilidad y mortalidad se ven aumentadas ${ }^{11}$.

En la actualidad se conocen más de 300 tipos diferentes de Betalactamasas ${ }^{2,12,13}$.

Las Betalactamasas de espectro extendido se ubican dentro de la clasificación de Bush y Jacoby en las categorías 2 be, 2 ber, 2 de y 2 der. Siendo una de sus características más importantes, desde el punto de vista epidemiológico, su capacidad de transferirse a través de bacterias de la misma o de diferente especie, por medio de elementos genéticos móviles, que favorece la diseminación de la resistencia bacteriana ${ }^{14,15}$.

Ante esto se plantea el objetivo de determinar la presencia de genes bla en aislados clínicos de Escherichia coli, en pacientes con infecciones de origen nosocomial provenientes del Hospital Nacional Zacamil, relacionándolo con el perfil de resistencia a los antimicrobianos utilizados en la atención de estas infecciones. Se espera que este estudio abra una ruta de investigación innovadora en El Salvador en cuanto al tema de Resistencia Bacteriana, para complementar los esfuerzos actuales en la investigación del fenómeno apoyándose en herramientas moleculares que permitan una mejor y más amplia comprensión del problema.

\section{METODOLOGÍA DE LA INVESTIGACIÓN}

Se analizaron cepas de Escherichia coli provenientes de muestras clínicas de diferentes sitios anatómicos de pacientes con infecciones intrahospitalarias, obtenida en los diferentes servicios de hospitalización del Hospital Nacional Zacamil, en la capital salvadoreña.

Las muestras se recolectaron durante los meses febrero a mayo del 2015 hasta obtener un total de 30 muestras. 
Se seleccionaron aislados consecutivos de Escherichia coli proveniente de muestras de pacientes con cuadro clínico compatible con Infección Asociada a la Atención en salud de origen intrahospitalario. Definiéndola como toda infección que no haya estado presente al momento del ingreso al hospital y que se haya desarrollado después de 48 horas de estancia hospitalaria ${ }^{11,16}$.

Se utilizó la cepa ATCC 25922 como cepa control según propone Clinical and Laboratory Standards Institute $2013^{17}$.

\section{Estudio de Concentración Mínima Inhibitoria (CMI)}

Se determinó la CMI de varios antibióticos según protocolo del Laboratorio Clínico del Hospital Zacamil, a partir del Sistema de Inoculación Prompt $^{\mathrm{TM}}-\mathrm{D}$ analizado con Sistema MicroScan ${ }^{\circledR}$.

La inoculación se llevó a cabo con el sistema RENOK $^{\circledR}$ con inoculadores D. La concentración final en cada pocillo fue de $3-7 \times 10^{5} \mathrm{UFC} / \mathrm{ml}$ según especificaciones del fabricante. Los paneles fueron incubados con el sistema WalkAway ${ }^{\circledR}$ y leídos a través del instrumento MicroScan ${ }^{\circledR}$ después de 18 a 20 horas de incubación, según especificaciones del fabricante. La CIM se registró como la concentración antimicrobiana más baja que muestra inhibición del crecimiento. Cuando hay crecimiento en todas las concentraciones de un antimicrobiano, la CIM se registra como "superior a" $(>)$ la concentración más alta. Cuando no hay crecimiento en ninguna de las concentraciones de los antimicrobianos, la CIM se registra como "menor o igual a" $(\leq)$ la concentración más baja.

\section{Determinación de BLEE}

A partir del reporte de resistencia y la producción de Betalactamasa de Espectro extendido para cada cepa obtenido por el sistema automatizado de estudio, se confirmó la producción de BLEE utilizando la técnica de difusión en agar con discos de antibióticos conteniendo cefalosporinas de tercera generación (ceftazidima y cefotaxima); sola y en combinación con ácido clavulánico como inhibidor de betalactamasa ${ }^{17}$.
Se realizó de forma similar al método convencional de difusión en agar MuellerHinton, con un inóculo Mac Farland 0,5 y ensayando 4 discos: ceftazidima, ceftazidima más ácido clavulánico, cefotaxima y cefotaxima más ácido clavulánico. Se incubó en atmósfera normal durante 18 horas a $37^{\circ} \mathrm{C}$. Posteriormente se midieron los halos de inhibición en forma convencional. El aumento del halo en más de $5 \mathrm{~mm}$ con los discos conteniendo la mezcla de cefalosporina y ácido clavulánico en relación al disco de cefalosporina sola, para las dos o al menos uno de las dos cefalosporinas se considera confirmatorio para la presencia de $\mathrm{BLEE}^{2,18,19}$.

\section{Reacción en cadena de polimerasa (PCR) para detección de genes bla.}

Para la detección de genes bla, todos los aislados clínicos fueron conservados en congelador a una temperatura de $-20^{\circ} \mathrm{C}$ hasta su procesamiento para la extracción de ADN bacteriano. Para la extracción de $\mathrm{ADN}$, se procedió a resucitar cada muestra sembrándola en caldo TSC e incubándolo a $37^{\circ} \mathrm{C}$ por 18 a 24 horas. Obteniendo cultivos recientes para la extracción del material genético. Para esto se utilizó KIT de extracción NucleoSpin ${ }^{\circledR}$ Tissue. Marca Macherey-Nagel. Realizando extracción según especificaciones del fabricante. El material obtenido, se distribuyó en alícuotas que fueron almacenadas a temperatura de $-20^{\circ} \mathrm{C}$ hasta su utilización para amplificación de genes bla. A todos los aislados se les realizó detección de genes $b l a_{\mathrm{CTX}-\mathrm{M} 1 / 2}, b l a_{\mathrm{TEM}} ; b l a_{\mathrm{SHV}} \mathrm{y}$ $b l a_{\text {AmpC }}$ mediante técnica molecular de PCR de punto final ${ }^{19}$. Los productos de PCR, fueron observados a través de geles de agarosa, teñidos con GelRedTM Nucleic Acid Gel Stain de la marca Biotium. Y observados con equipo de fotocaptura DigiDoc- $\mathrm{It}^{\circledR}$.

Los partidores utilizados para la detección de estos genes bla fueron los siguientes ${ }^{14,20,21,22}$.

Para la amplificación de estos genes, se siguieron las siguientes condiciones de temperaturas realizando 35 ciclos de amplificación en Termociclador: 


\begin{tabular}{|c|c|c|c|}
\hline Gen $b l a$ & Secuencia & Tamaño pb & Referencia \\
\hline$B l a_{\mathrm{TEM}}$ & $\begin{array}{l}\text { 5’ TGGGTGCACGAGTGGGTTAC 3’ } \\
\text { 5' TTATCCGCCTCCATCCAGTC 3' }\end{array}$ & 526 & Tenover. $(1994)^{20}$ \\
\hline$B l a_{\mathrm{SHV}}$ & $\begin{array}{l}\text { 5’ CTGGGGAAACGGAACTGAAATG 3' } \\
\text { 5’ GGGGTATCCCGCAGATAAAT 3' }\end{array}$ & 389 & Bello $(2005)^{14}$ \\
\hline$b l a_{\mathrm{CTX}-\mathrm{M}}$ & $\begin{array}{l}\text { 5'-TCAAAACTGGCAGCCG-3' } \\
\text { 5'ACCGCGATATCGTTGGT 3' }\end{array}$ & 551 & Bonnet $(2000)^{21}$ \\
\hline$b l a_{\mathrm{AmpC}}$ & $\begin{array}{l}\text { 5'-TCAAAACTGGCAGCCG-3' } \\
\text { 5'-GAGCCCGTTTTATGCACCCA-3 }\end{array}$ & 311 & Paterson $(2003)^{22}$ \\
\hline
\end{tabular}

Temperatura de denaturalización de $94^{\circ} \mathrm{C}$ por 30 segundos. Temperatura de alineamiento de $60^{\circ} \mathrm{C}$ por 1 minuto $\left(70^{\circ} \mathrm{C}\right.$ para CTX-M1/2 y $45^{\circ} \mathrm{C}$ para TEM). Temperatura de extensión de $72^{\circ} \mathrm{C}$ por 1 minuto. Seguido de una Extensión final de $72^{\circ} \mathrm{C}$ por $10 \mathrm{~min}^{22}$.

\section{RESULTADOS}

Se recolectaron 30 muestras de diferentes sitios anatómicos, provenientes de pacientes ingresados en los diferentes servicios de encamados del Hospital Nacional Zacamil, siendo la muestra más frecuente, orina. Estas muestras corresponden a infecciones asociadas a la atención en salud y fueron recolectadas en el periodo de febrero a mayo de 2015.

Se procesaron en el Laboratorio de Microbiología de la Universidad Evangélica de El Salvador, en el periodo de marzo a junio de 2015 para la determinación de BLEE por método de doble disco. Posteriormente fueron almacenadas en congelador a temperatura de $-20^{\circ} \mathrm{C}$ hasta su procesamiento para extracción de ADN y posterior amplificación de genes para BLEE.

Todas las muestras recolectadas reflejan valores de CIM, correspondientes a resistencia a por lo menos un antibiótico betalactámico. Esto puede observarse en la Tabla $N^{\circ} 1$ y 2 correspondientes a los valores de CIM de los antibióticos analizados, observándose que la mayoría de muestras presentan disminución de la susceptibilidad a cefalosporinas de tercera generación como ceftriazona y cefotaxima. También se determinó susceptibilidad disminuida para la combinación trimetroprim/sulfametoxazol en la mayoría de los aislados estudiados. 
Tabla 1. Valores CIM de diferentes aislados clínicos de Escherichia coli para los antibióticos estudiados.

\begin{tabular}{|c|c|c|c|c|c|c|c|c|c|c|c|c|c|}
\hline Cepa & AMC & SAM & AMP & ATM & CEP & $\mathrm{CZO}$ & FEP & CTX & CTT & FOX & CAZ & $\mathrm{CRO}$ & CXM \\
\hline EC-04 & $\leq 8 / 4$ & $>16 / 8$ & $>16$ & $>16$ & $>16$ & $>16$ & $>16$ & $>32$ & $\leq 16$ & $\leq 8$ & $>16$ & $>32$ & $>16$ \\
\hline EC-05 & $16 / 8$ & $>16 / 8$ & $>16$ & $>16$ & $>16$ & $>16$ & $>16$ & $>32$ & $\leq 16$ & $\leq 8$ & $>16$ & $>32$ & $>16$ \\
\hline EC-08 & $\leq 8 / 4$ & $16 / 8$ & $>16$ & $\leq 8$ & $>16$ & $\leq 8$ & $\leq 8$ & $\leq 2$ & $\leq 16$ & $\leq 8$ & $\leq 1$ & $\leq 8$ & 8 \\
\hline EC-09 & $\leq 8 / 4$ & $\leq 8 / 4$ & $\leq 8$ & $\leq 8$ & 16 & $\leq 8$ & $\leq 8$ & $\leq 2$ & $\leq 16$ & $\leq 8$ & $\leq 1$ & $\leq 8$ & $\leq 4$ \\
\hline EC-11 & $16 / 8$ & $>16 / 8$ & $>16$ & $\leq 8$ & 16 & $\leq 8$ & $\leq 8$ & $\leq 2$ & $\leq 16$ & $\leq 8$ & $\leq 1$ & $\leq 8$ & $\leq 4$ \\
\hline EC-12 & $>16 / 8$ & $>16 / 8$ & $>16$ & $>16$ & $>16$ & $>16$ & $>16$ & $>32$ & $\leq 16$ & $\leq 8$ & $>16$ & $>32$ & $>16$ \\
\hline EC-14 & $16 / 8$ & $>16 / 8$ & $>16$ & $>16$ & $>16$ & $>16$ & $>16$ & $>32$ & $\leq 16$ & $\leq 8$ & $>16$ & $>32$ & $>16$ \\
\hline EC-17 & $>16 / 8$ & $16 / 8$ & $>16$ & $>16$ & $>16$ & $>16$ & $>16$ & $>32$ & $\leq 16$ & $\leq 8$ & $>16$ & $>32$ & $>16$ \\
\hline EC-20 & $\leq 8 / 4$ & $>16 / 8$ & $>16$ & $>16$ & $>16$ & $>16$ & $<16$ & $>32$ & $\leq 16$ & $\leq 8$ & $>16$ & $>32$ & $>16$ \\
\hline EC-23 & $16 / 8$ & $>16 / 8$ & $>16$ & $>16$ & $>16$ & $>16$ & $>16$ & $>32$ & $\leq 16$ & $\leq 8$ & $>16$ & $>32$ & $>16$ \\
\hline EC-26 & $16 / 8$ & $>16 / 8$ & $>16$ & $>16$ & $>16$ & $>16$ & $>16$ & $>32$ & $\leq 16$ & $\leq 8$ & $>16$ & $>32$ & $>16$ \\
\hline EC-27 & $>16 / 8$ & $>16 / 8$ & $>16$ & $>16$ & $>16$ & $>16$ & $>16$ & $>32$ & $\leq 16$ & $\leq 8$ & $>16$ & $>32$ & $>16$ \\
\hline EC-30 & $16 / 8$ & $>16 / 8$ & $>16$ & $\leq 8$ & 16 & $\leq 8$ & $\leq 8$ & $\leq 2$ & $\leq 16$ & $\leq 8$ & $\leq 1$ & $\leq 8$ & $\leq 4$ \\
\hline EC-31 & $\leq 8 / 4$ & $>16 / 8$ & $>16$ & $\leq 8$ & 16 & $\leq 8$ & $\leq 8$ & $\leq 2$ & $\leq 16$ & $\leq 8$ & $\leq 1$ & $\leq 8$ & $\leq 4$ \\
\hline EC-32 & $16 / 8$ & $>16 / 8$ & $>16$ & $>16$ & $>16$ & $>16$ & $>16$ & $>32$ & $\leq 16$ & 16 & $>16$ & $>32$ & $>16$ \\
\hline EC-33 & $\leq 8 / 4$ & $>16 / 8$ & $>16$ & $\leq 8$ & 16 & $\leq 8$ & $\leq 8$ & $\leq 2$ & $\leq 16$ & $\leq$ & $\leq 1$ & $\leq 8$ & $\leq$ \\
\hline EC-34 & $16 / 8$ & $>16 / 8$ & $>16$ & $>16$ & $>16$ & $>16$ & $>16$ & $>32$ & $\leq 16$ & $\leq 8$ & $>16$ & $>32$ & $>16$ \\
\hline EC-35 & $\leq 8 / 4$ & $>16 / 8$ & $>16$ & $>16$ & $>16$ & $>16$ & $>16$ & $>32$ & $\leq 16$ & $\leq 8$ & $>16$ & $>32$ & $>16$ \\
\hline EC-36 & $>16 / 8$ & $>16 / 8$ & $>16$ & $>16$ & $>16$ & $>16$ & $>16$ & $>32$ & $\leq 16$ & 16 & $>16$ & $>32$ & $>16$ \\
\hline EC-37 & $\leq 8 / 4$ & $>16 / 8$ & $>16$ & $>16$ & $>16$ & $>16$ & $>16$ & $>32$ & $\leq 16$ & $\leq 8$ & $>16$ & $>32$ & $>16$ \\
\hline EC-39 & $16 / 8$ & $>16 / 8$ & $>16$ & $>16$ & $>16$ & $>16$ & $>16$ & $>32$ & $\leq 16$ & $\leq 8$ & $>16$ & $>32$ & $>16$ \\
\hline EC-40 & $16 / 8$ & $>16 / 8$ & $>16$ & $>16$ & $>16$ & $>16$ & $>16$ & $>32$ & $\leq 16$ & $\leq 8$ & $>16$ & $>32$ & $>16$ \\
\hline EC-41 & $16 / 8$ & $>16 / 8$ & $>16$ & $>16$ & $>16$ & $>16$ & $>16$ & $>32$ & $\leq 16$ & $\leq 8$ & $>16$ & $>32$ & $>16$ \\
\hline EC-42 & $16 / 8$ & $>16 / 8$ & $>16$ & $>16$ & $>16$ & $>16$ & $>16$ & $>32$ & $\leq 16$ & 16 & $>16$ & $>32$ & $>16$ \\
\hline EC-43 & $16 / 8$ & $>16 / 8$ & $>16$ & $>16$ & $>16$ & $>16$ & $>16$ & $>32$ & $\leq 16$ & 16 & $>16$ & $>32$ & $>16$ \\
\hline EC-44 & $16 / 8$ & $>16 / 8$ & $>16$ & $\leq 8$ & 16 & $\leq 8$ & $\leq 8$ & $\leq 2$ & $\leq 16$ & $\leq 8$ & $\leq 1$ & $\leq 8$ & 8 \\
\hline EC-46 & $\leq 8 / 4$ & $16 / 8$ & $>16$ & $\leq 8$ & $>16$ & $\leq 8$ & $\leq 8$ & $\leq 25$ & $\leq 16$ & $\leq 8$ & $\leq 1$ & $\leq 8$ & $\leq 4$ \\
\hline EC-48 & $\leq 8 / 4$ & $16 / 8$ & $>16$ & $\leq 8$ & $>16$ & $\leq 8$ & $\leq 8$ & $\leq 2$ & $\leq 16$ & $\leq 8$ & $\leq 1$ & $\leq 8$ & 8 \\
\hline EC-49 & $\leq 8 / 4$ & $16 / 8$ & $>16$ & $\leq 8$ & 16 & $\leq 8$ & $\leq 8$ & $\leq 2$ & $\leq 16$ & $\leq 8$ & 4 & $\leq 8$ & $\leq 4$ \\
\hline EC-51 & $\leq 8 / 4$ & $>16 / 8$ & $<16$ & $\leq 8$ & $>16$ & $\leq 8$ & $\leq 8$ & $\leq 2$ & $\leq 16$ & $\leq 8$ & $\leq 1$ & $\leq 8$ & $\leq 4$ \\
\hline
\end{tabular}

AMC: amoxicilina/ac.clav; SAM: ampicilina/sulbactam; AMP: ampicilina; ATM: aztreonam; CEP: cefalotina; CZO: cefazolina; FEP: cefepime; CTX: cefotaxima; CTX: cefotaxima; CTT: cefotetan; FOX:cefoxitina; CAZ: ceftazidima; CRO: ceftriazona; CXM: cefuroxima

Es importante mencionar que la mayoría de aislados estudiados presentaban CIM a nitrofurantoina por debajo de los puntos de corte, correspondiente a susceptibilidad, como puede observarse en la Tabla 2. 
Tabla 2. Resultado valores de CIM 50 y 90 de los antibióticos estudiados para los diferentes aislados clínicos de Escherichia coli.

\begin{tabular}{|c|c|c|c|c|c|c|}
\hline \multirow[b]{2}{*}{ Antibiotico } & \multirow[b]{2}{*}{ Rango } & \multirow{2}{*}{$\begin{array}{c}\mathrm{CIM}(\mu g r / m l) \\
50 \%\end{array}$} & \multirow[b]{2}{*}{$90 \%$} & \multicolumn{3}{|c|}{ Puntos de corte } \\
\hline & & & & $S$ & $\mathrm{I}$ & $\mathrm{R}$ \\
\hline AMK & $\leq 16$ a $>32$ & $\leq 16$ & 32 & $\leq 16$ & 32 & $\geq 64$ \\
\hline AMC & $\leq 8 / 4$ a $>16 / 8$ & $\leq 8 / 4$ & $>16 / 8$ & $\leq 8 / 4$ & $16 / 8$ & $\geq 32 / 16$ \\
\hline SAM & $\leq 8 / 4$ a $>16 / 8$ & $>16 / 8$ & $>16 / 8$ & $\leq 8 / 4$ & $16 / 8$ & $\geq 32 / 16$ \\
\hline AMP & $\leq 8 \mathrm{a}>16$ & $>16$ & $>16$ & $\leq 8$ & 16 & $\geq 32$ \\
\hline ATM & $\leq 8 \mathrm{a}>16$ & $>16$ & $>16$ & $\leq 4$ & 8 & $\geq 16$ \\
\hline CEP & $\leq 8 \mathrm{a}>16$ & $>16$ & $>16$ & $\leq 8$ & 16 & $\geq 32$ \\
\hline $\mathrm{CZO}$ & $\leq 8 \mathrm{a}>16$ & $>16$ & $>16$ & $\leq 1$ & 2 & $\geq 4$ \\
\hline FEP & $\leq 8 \mathrm{a}>16$ & $>16$ & $>16$ & $\leq 8$ & 13 & $\geq 32$ \\
\hline CTX & $\leq 2 \mathrm{a}>32$ & $>32$ & $>32$ & $\leq 1$ & 2 & $\geq 4$ \\
\hline $\mathrm{CAC}$ & $\leq 0.5 \mathrm{a}>4$ & $\leq 0.5$ & $\leq 0.5$ & Test de & BLEE & \\
\hline CTT & $\leq 16$ a $>32$ & $\leq 16$ & $\leq 16$ & $\leq 16$ & 32 & $\geq 64$ \\
\hline FOX & $\leq 8 \mathrm{a}>16$ & $\leq 8$ & 16 & $\leq 8$ & 16 & $\geq 32$ \\
\hline CAZ & $\leq 1 \mathrm{a}>16$ & $>16$ & $\leq 16$ & $\leq 4$ & 8 & $\geq 16$ \\
\hline CAC & $\leq 0.25 \mathrm{a}>2$ & $\leq 0.25$ & $>2$ & Test de & BLEE & \\
\hline CRO & $\leq 8 \mathrm{a}>32$ & $>32$ & $>32$ & $\leq 1$ & 2 & $\geq 4$ \\
\hline CXM & $\leq 4 \mathrm{a}>16$ & $>16$ & $>16$ & $\leq 8$ & 16 & $\geq 32$ \\
\hline CIP & $\leq 1 \mathrm{a}>2$ & $>2$ & $>2$ & $\leq 1$ & 2 & $\geq 4$ \\
\hline EPM & $\leq 2 \mathrm{a}>4$ & $\leq 2$ & $\leq 2$ & $\leq 0.25$ & 0.5 & $\geq 1$ \\
\hline GEN & $\leq 4 \mathrm{a}>8$ & $>8$ & $>8$ & $\leq 4$ & 8 & $\geq 16$ \\
\hline IMP & $\leq 4$ & $\leq 4$ & $\leq 4$ & $\leq 1$ & 2 & $\geq 4$ \\
\hline LEV & $\leq 2 \mathrm{a}>4$ & $>4$ & $>4$ & $\leq 2$ & 4 & $\geq 8$ \\
\hline MER & $\leq 4$ & $\leq 4$ & $\leq 4$ & $\leq 1$ & 2 & $\geq 4$ \\
\hline MOX & $\leq 2 \mathrm{a}>4$ & $>4$ & $>4$ & ND & ND & $\mathrm{ND}$ \\
\hline NIT & $\leq 32 \mathrm{a}>64$ & $\leq 32$ & $\leq 32$ & $\leq 32$ & 64 & $\geq 128$ \\
\hline PIC & $\leq 16 \mathrm{a}>64$ & $\leq 16$ & 64 & $\leq 16 / 4$ & $32 / 4-64 / 4$ & $\geq 128 / 4$ \\
\hline TET & $\leq 4 \mathrm{a}>8$ & $>8$ & $>8$ & $\leq 4$ & 8 & $\geq 16$ \\
\hline TAC & $\leq 16$ a $>64$ & 64 & 64 & $\leq 16 / 2$ & $32 / 2-64 / 2$ & $\geq 128 / 2$ \\
\hline TOB & $\leq 8 \mathrm{a}>8$ & $>8$ & $>8$ & $\leq 4$ & 8 & $\geq 16$ \\
\hline $\mathrm{COX}$ & $\leq 2 / 38 \mathrm{a}>2 / 38$ & $>2 / 38$ & $>2 / 38$ & $\leq 2 / 38$ & ND & $\geq 4 / 76$ \\
\hline
\end{tabular}

AMK: amikacina; AMC: amoxicilina/ac.clav; SAM: ampicilina/sulbactam; AMP: ampicilina; ATM: aztreonam; CEP: cefalotina; CZO: cefazolina

FEP: cefepime; CTX: cefotaxima; CTC: cefotaxima/ac.clav; CTT: cefotetan; FOX:cefoxitina; CAZ: ceftazidima; CAC: ceftazidima/ac. clav; CRO: ceftriazona

CRO:ceftriazona; CXM:cefuroxima; CIP:ciprofloxacina; EPM:ertapenem; GEN:gentamicina; IMP:imipenem; LEV: levofloxacina; MER: meropenem

MOX:moxifloxacina; NIT:nitrofurantoina; PIC:piperacilina/tazobactam; TET:tetraciclina; TAC: ticarcilina/ac.clav; TOB: tobramicina; COX: Trim/sulfam. 
La determinación de BLEE varió entre el método automatizado y el test de doble disco. Se realizó el ensayo de doble disco en todos los aislados clínicos, encontrándose mayor positividad al realizar la prueba manualmente (Figura 1).

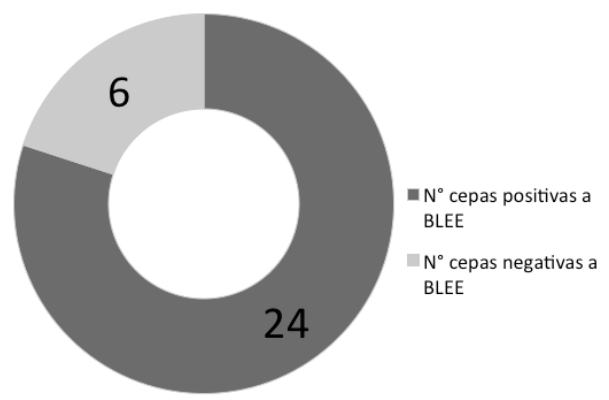

Figura 1. Distribución de cepas productoras de BLEE a partir de resultado test de doble disco.

Por método fenotípico de difusión en agar con doble disco, se obtuvo confirmación de producción de enzimas en el $80 \%$ de las muestras analizadas. Cabe mencionar que al revisar el reporte de producción de BLEE por método automatizado, este solamente detectó la producción de la enzima en el $66 \%$ de las muestras.

Se relacionó estadísticamente el resultado de producción de BLEE determinado por el método automatizado del Hospital y por el método de doble disco realizado en el Laboratorio de la Universidad Evangélica de El Salvador. Para ello se calculó CHI cuadrada, a través del paquete estadístico de EXCEL 2010, utilizándose un valor de $\mathrm{p}<0.05$. Al realizar la prueba de X2 el valor de $\mathrm{p}$ fue 0.00000293 para el test de doble disco. Este valor confirma la significancia de los resultados de la prueba (Figura 2).

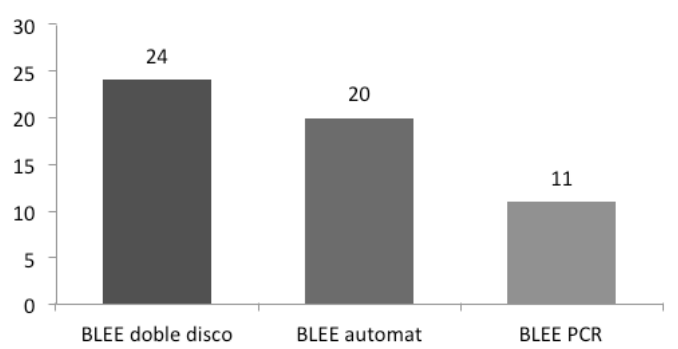

Figura 2. Muestras que resultaron positivas a identificación de BLEE por los diferentes métodos utilizados.
Es importante mencionar que la determinación de BLEE resultó positiva únicamente a la enzima tipo TEM. Sin encontrar positividad a los genes de las otras tres enzimas estudiadas.

Al revisar los 30 expedientes clínicos de pacientes de los cuales se obtuvo las muestras, se verificó que la mayoría recibieron tratamiento con al menos un antibiótico reportado como resistente por el Laboratorio de Bacteriología del Hospital. $\mathrm{Y}$ en varios casos se continuó con la terapia aun después de conocido el resultado del cultivo.

El antibiótico que se utilizó con mayor frecuencia para el tratamiento de infecciones resistentes fue ciprofloxacina, seguido de ceftriazona. Algunos de los pacientes incluso recibieron terapia combinada de antibióticos, utilizando dos y hasta tres medicamentos simultáneamente a los cuales el cultivo reportaba el microorganismo aislado como resistente (Tabla 3 ).

Tabla 3. Frecuencia de pacientes que recibió tratamiento empírico por cada uno de los antibióticos propuestos por el establecimiento.

\begin{tabular}{|c|c|}
\hline Antibiótico & Pacientes \\
\hline AMP & 2 \\
PEN & 1 \\
AMX & 1 \\
AMK & 2 \\
DIC & 1 \\
FOS & 1 \\
CRO & 8 \\
CZO & 1 \\
CIP & 12 \\
MTZ & 4 \\
CLI & 4 \\
DOR & 1 \\
NIT & 2 \\
COX & 2 \\
CHL & 1 \\
\hline
\end{tabular}

AMP: ampicilina; PEN: penicilina; AMX: amoxicilina; AMK: amikacina; DIC: dicloxacilina FOS: fosfomicina ; CRO: ceftriazona; CZO: cefazolina; CIP: ciprofloxacina; MTZ: metronidazol ;CLI: clindamicina; DOR: doripenem; NIT: nitrofurantoina; COX: trimetroprim/sulfametoxazol; CHL: cloranfenicol. 
Del total de pacientes con IAAS de los cuales se obtuvieron las muestras, solamente dos fueron tratados con Nitrofurantoina durante el curso de su enfermedad, a pesar que en la mayoría de aislados se reportaban niveles de susceptibilidad a este fármaco (Figura 3).

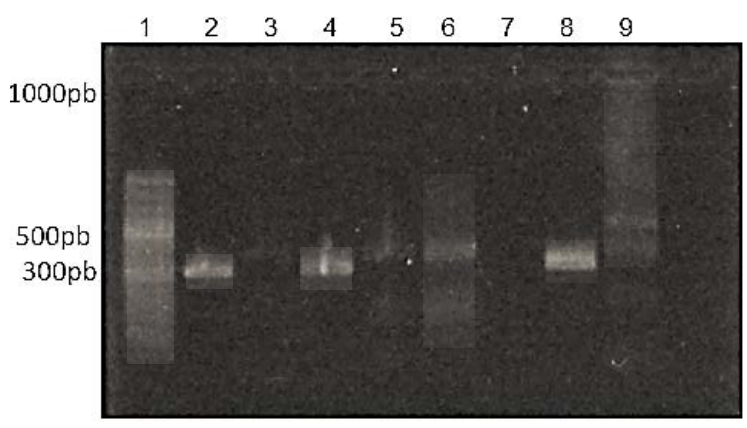

Figura 3. Electroforesis de productos de PCR del gen blaTEM. Carril 1: marcador de peso molecular de 1000 pb plus; 2: control positivo; 3: EC-32; 4: EC-33; 5: EC-36; 6: EC-39; 7: EC-40; 8: EC-41; 9: EC-42.

En once de las muestras estudiadas se pudo constatar la presencia de genes bla tipo TEM, siendo el único de los cuatro genes estudiados que pudo amplificarse (Figura 4).

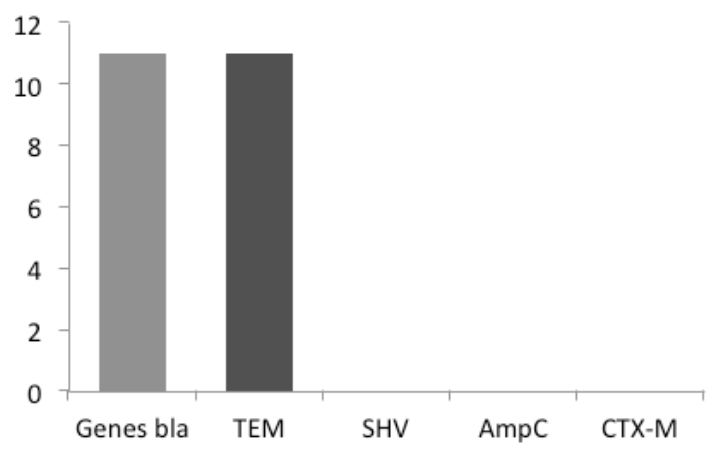

Figura 4. Presencia de genes bla en aislados de Escherichia coli.

\section{DISCUSIÓN}

Las infecciones asociadas a la atención en salud son un verdadero problema de salud pública por los costos humanos y económicos que representan. Y si estas IAAS son ocasionadas por microorganismos resistentes, el problema se agrava, llegando en algunos casos a duplicar el tiempo de internamiento y la mortalidad asociada.

Para un país como El Salvador es prioritario implementar medidas de control de infecciones intrahospitalarias. Y sobre todo, de diseminación de factores de resistencia bacteriana, ya que el costo económico asociado a la atención de las mismas representa un impacto significativo en los recursos destinados a la atención en salud.

Una de las estrategias principales para el control de la resistencia bacteriana es el conocimiento e investigación de los principales microorganismos asociados a infecciones de origen nosocomial de cada establecimiento. Existen diversos mecanismos de resistencia y una especie bacteriana puede expresar uno o varios de esos mecanismos. Conocer las características microbiológicas de estos agentes bacterianos asociados a IAAS, proporciona a cada establecimiento mejores bases para la comprensión del fenómeno de resistencia y por ende mejores estrategias de control y prevención.

En la actualidad en El Salvador existen muy pocos estudios referentes a las características microbiológicas de los agentes resistentes asociados a IAAS de los principales nosocomios del país. Y los estudios hasta la fecha publicados, enfocan el problema desde una perspectiva fenotípica únicamente.

En este estudio se utiliza una estrategia de laboratorio que en El Salvador resulta innovadora, ya que es la primera vez que se aplica metodología de PCR para la investigación del fenómeno de la resistencia bacteriana. Esto proporciona una perspectiva más amplia del fenómeno, pues permite iniciar una nueva ruta de investigación que oriente, no solo a la prevención de la resistencia observada o expresada por el microorganismo en su contacto con el antimicrobiano, sino a la prevención considerando la presión selectiva sobre el genoma bacteriano. Reduciendo así posibilidad de transferencia de genes de resistencia intra e inter especies. 
En la actualidad, poco se consideran las características genéticas del agente bacteriano a la hora de decidir una terapia. El clínico se decide por terapias empíricas que han demostrado efectividad en el control de la infección, aunque esto suponga ejercer mayor presión selectiva sobre los microorganismos, favoreciendo el aparecimiento de multiresistencia. Lo anterior puede constatarse en los resultados obtenidos de este estudio, donde la mayoría de aislados obtenidos presentaba resistencia a cefalosporinas de tercera generación como ceftriazona. Siendo este último uno de los medicamentos que más se utilizó para el tratamiento de las infecciones identificadas.

Con esta investigación pudimos comprobar que, en la mayoría de los pacientes con infecciones por microorganismos resistentes, se utilizó al menos un medicamento que durante las pruebas de susceptibilidad presentó valores de resistencia.

Otro elemento importante de mencionar es la frecuente utilización de ciprofloxacina como terapia para infecciones del tracto urinario, a pesar que la mayoría de aislados presentaban resistencia a este medicamento. Además de su conocido efecto en la selección de cepas productoras de $\mathrm{BLEE}^{22,23}$.

Por otra parte, muchos de estos pacientes con infecciones resistentes fueron tratados con protocolos de tratamientos en combinación de antibióticos. Estas combinaciones muchas veces resultaron en éxito terapéutico. Pues frecuentemente, en la elección de los medicamentos se incluyó al menos un antibiótico para el cual el microorganismo era susceptible.

A pesar de que el Hospital dispone de Laboratorio de Bacteriología para la realización de pruebas rutinarias de susceptibilidad microbiana a través de métodos automatizados, estos resultados no siempre se toman en consideración a la hora de decidir la terapéutica a utilizar. En ocasiones el clínico instaura terapias basado en consideraciones clínicas y su propia experiencia. Esto puede estar influenciado por la disponibilidad de los medicamentos con los que cuenta el establecimiento, siendo los medicamentos que se eligen las únicas opciones con las que se cuentan en el momento de atender el paciente.

Consideración aparte merece el uso de nitrofurantoina, medicamento de amplio espectro utilizado para el tratamiento de infecciones del tracto urinario no complicado de origen comunitario, el cual interfiere con el metabolismo bacteriano de forma eficaz. En este estudio, resultó que el $90 \%$ de los aislados presentaba valores de CMI por debajo de los puntos de corte establecidos como susceptibilidad. Sin embargo solo fue utilizado en dos de los pacientes estudiados. Además es un medicamento de menor costo que las cefalosporinas de tercera generación, y de administración oral, permite al paciente completar su tratamiento de forma ambulatoria, reduciendo su periodo de internamiento; y por tanto, los costos de atención. Siempre y cuando no se presenten otras condiciones clínicas que requieran de vigilancia intrahospitalaria. Claro está que esta valoración debe ser realizada por el Médico Tratante, tomando en cuenta las condiciones clínicas individuales de cada paciente.

Por otro lado, se debe considerar que la producción de enzimas inactivantes es uno de los mecanismos de resistencia más ampliamente reportados a nivel mundial. Siendo las BLEE de mayor relevancia por el amplio perfil de resistencia que exhiben las bacterias que las producen. Existen más de 300 tipos diferentes de BLEE, algunas de ellas más frecuentes en ciertas áreas geográficas. A pesar que en la actualidad existen múltiples reportes de microorganismos resistentes asociados a la producción de estas enzimas, en El Salvador, no existe ningún reporte previo que identifique el tipo de enzima que un microorganismo produce para la inactivación antibiótica.

En este estudio se pudo aislar microorganismos productores de BLEE identificados como Escherichia coli, en los que se determinó 
por métodos fenotípicos y moleculares la producción de BLEE tipo TEM. Un grupo de Betalactamasas ampliamente diseminadas en el mundo y que frecuentemente se asocian a E.coli multiresistente. Fueron identificadas por primera vez en 1963, en Grecia, en un plásmido de una cepa de E. coli obtenida de una niña de nombre Temoniera, de ahí su nombre. Pertenece al grupo $2 \mathrm{~b}$ de Betalactamasas ${ }^{24,25}$. En la actualidad se reconocen alrededor de 200 enzimas distintas de este tipo, algunas de ellas reconocidas como de amplio espectro. Poseen una mayor afinidad por CAZ que por CTX, evidente, por los niveles de resistencia de estos antibióticos ${ }^{26}$. Esta enzima se encuentra frecuentemente en cepas de Escherichia coli resistente a carbapenémicos.

Dentro de este grupo de BLEE se encuentran algunas que tienen resistencia para inhibidores de Betalactamasas y que presentar mayor capacidad hidrolítica sobre cefalosporinas de tercera generación como ceftazidima. Esto puede explicar los resultados presentes de CMI en los aislados clínicos obtenidos.

Es importante mencionar que los genes que codifican para esta enzima tipo TEM frecuentemente pueden encontrarse en elementos móviles del genoma bacteriano, facilitando su transferencia a otras especies bacterianas e inclusos a géneros bacterianos diferentes.

A pesar que se intentó la amplificación de otros tres genes diferentes de BLEE, no fue posible, probablemente debido a la posibilidad de estar ante un clon de E. coli que exhiba el mismo patrón genético. Aunque no pudo ser comprobado en este estudio, no significa que puedan encontrarse genes para otras Betalactamasas diferentes a las estudiadas.

La utilización de herramientas moleculares de estudio de la resistencia bacteriana abre una puerta de posibilidades que permiten un mejor conocimiento, basado en la investigación del fenómeno desde una perspectiva más amplia, nunca antes abordado en El Salvador. Es necesario que las instituciones encargadas de estudiar y promover el adecuado uso de los antibióticos y de la prevención de la resistencia bacteriana, destinen mayores recursos que permitan más y mejores investigaciones utilizando este tipo de herramientas moleculares.

\section{CONCLUSIONES}

- La mayoría de aislados clínicos resultaron positivos a producción de BLEE determinado por cualquiera de los tres métodos utilizados para su identificación.

- El método de difusión de doble disco en agar resultó más eficaz para determinar la producción de BLEE.

- Ceftriazona y ciprofloxacina fueron los antibióticos más utilizados para el tratamiento de infecciones ocasionadas por Escherichia coli de origen intrahospitalario, a pesar que la mayoría de aislados resultaron resistentes a estos medicamentos.

- La mayoría de aislados clínicos de Escherichia coli estudiados resultaron susceptibles a nitrofurantoina.

- Se determinó la presencia de genes bla tipo TEM en el 36\% de las muestras estudiadas.

\section{AGRADECIMIENTOS}

A Universidad Evangélica de El Salvador por el apoyo financiero para la investigación.

A Johanna Sanoja, asesora científica de Scientific
Instrument, por su apoyo técnico para la
realización de esta investigación.

A la licenciada Jacqueline de Arias por su esfuerzo y apoyo en la implementación del Laboratorio de Biología Molecular. 


\section{REFERENCIAS BIBLIOGRÁFICAS}

1. Blair JM, Weeber M, Baylay A, Ogbolu D y Piddock L. Molecular Mechanisms of Antibiotic resistance. Nat Rev Microbiol, 2014; 13: 42-51.

2. García Hernández A, García Vásquez E, Hernández Torres A, Ruíz J, Yagüe G, Herrero JA, Gómez J. Bacteriemias por Escherichia coli productor de betalactamasas de espectro extendido (BLEE): significación clínica y perspectivas actuales. Rev Esp Quimioter 2011; 24(2): 57-66.

3. Cisneros J, Cordero E. Relevancia de las BLEE en el Pronóstico y Coste de las Infecciones. Enferm Infecc Microbiol Clin. 2007; 25(2): 48-53.

4. Rodríguez-Baño J, Navarro MD, Romero L, MartínezMartínez L, Muniain MA, Perea EJ, Pascual A. Epidemiology and clinical features of infections caused by extended.spectrum $ß$-lactamase producing Escherichia coli in non hospitalized patients. J Clin Microbiol 2004;42: 1089-94.

5. García C, Astocondor L, Banda C. Enterobacterias productoras de $\beta$-lactamasas de espectro extendido: Situación en América Latina y el Perú. Acta Med Per 2012;29(3): 163-169.

6. Oteo J. Antibiotic resistance in 1962 invasive isolates of Escherichia coli in 27 Spanish hospitals participating in the European Antimicrobial Resistance Surveillance System (2001). J Antimicrob Chemother 2002;50: 945-52.

7. Oteo J, Pérez-Vázquez M, Campos J. Extended-spectrum [beta]lactamase producing Escherichia coli: changing epidemiology and clinical impact. Curr Opin Infect Dis 2010;23: 320-6.

8. Vasquez A, Montero $M$, Laughlin M, Dancy E, Melmed R, Sosa L, Watkins L, Folster J, Strockbine N, Moulton-Meissner H, Ansari U, Cartter M, Spalding Walters M. Investigation of Escherichia coli Harboring the mcr-1 Resistance Gene - Connecticut. MMWR 2016; 65 (36): 979 - 980.

9. Jayol A, Poirel L, Dortet L, Nordmann P. National survey of colistin resistance among carbapenemase-producing Enterobacteriaceae and outbreak caused by colistin-resistant OXA-48-producing Klebsiella pneumoniae, France, 2014. Euro Surveill. 2016; 21(37): 30339.
10. Flores MK, Perez LM, Trelles MG, Malaga M, Loza M, Tapia E. Infección urinaria intrahospitalaria en los servicios de hospitalización de Medicina de un hospital general. Rev Med Hered 2008; 19 (2): 46-52.

11. Rubtsova M Y, Ulyashova M M, Bachmman T T, Schmid R D \& Egorov A M. Multiparametric Determination of Genes and their Point Mutations for Identification of Beta-Lactamases. Biochemistry 2010; 75 (13): 1628.

12. Thenmozhi S, Moorthy K, Sureshkumar BT, Suresh M. Antibiotic Resistance Mechanism of ESBL Producing Enterobacteriaceae in Clinical Field: A Review. Int. J. Pure App. Biosci. 2014; 2(3): 207-226.

13. Jafari A, Aslani M, Bouzari S. Escherichia coli: a brief review of diarrheagenic pathotypes and their role in diarrheal diseases in Iran. IRAN. J. MICROBIOL. 2012; 4(3): 102-117.

14. Bryce A, Costelloe C, Hawcroft C, Wootton M, Hay A. Faecal carriage of antibiotic resistant Escherichia coli in asymptomatic children and associations with primary care antibiotic prescribing: a systematic review and meta-analysis. BMC Infectious Diseases 2016; 16: 359 .

15. Bello $H$. Bases genéticas de la resistencia a cefalosporinas de tercera generación en cepas de Klebsiella pneumoniae subespecie pneumoniae aisladas de hospitales chilenos. Tesis para optar al grado de Doctor en Ciencias Biológicas, área Biología Celular y Molecular, Universidad de Concepción, Chile 2005. 175pp.

16. Paterson DL, Mulazimoglu L, Casellas JM, Ko WC, Goossens H, von Gottberg A, et al. Epidemiology of ciprofloxacin resistance and its relationship to extended-spectrum $\beta$-lactamase production in Klebsiellla pneumoniae isolates causing bacteremia. Clin Infect Dis. 2000; 30(3): 473-8.

17. Horan T, Andrus M, Dudeck M. CDC/NHSN surveillance definition of health care-associated infection and criteria for specific types of infections in the acute care setting. Am J Infect Control 2008; 36: 309-332.

18. Clinical and Laboratory Standards Institute. Methods for Dilution Antimicrobial Susceptibility Tests for Bacteria That Grow Aerobically; Approved Standard. Ninth Edition. CLSI document M07-A9. (ISBN 1-56238-783-9). Clinical and Laboratory Standard Institute, 2012, Wayne, Pensylvania, USA. 
19. Zemelman $\mathrm{R}$, Valenzuela $\mathrm{L}$, Domínguez $\mathrm{M}$, Bello H, González G, Zemelman C. Detección de Betalactamasas de Espectro Extendido en el Laboratorio de Microbiología. Rev Chil Infect, 2002; 19(2): 592-595.

20. Gaitán SL, Espinal P y Grupo de Investigación en Resistencia, Región Caribe. Caracterización molecular de Escherichia coli y Klebsiella pneumoniae productores de $\beta$ - lactamasa de espectro extendido en hospitales de la Región Caribe, Colombia. Rev Chil de Infect, 2009; 26(3): 239-246.

21. Tenover FC, Huang Ming B, Rasheed JK y Persing DH. Development of PCR Assays To Detect Ampicillin Resistance Genes in Cerebrospinal Fluid Samples Containing Haemophilus influenza. J Clin Microbiol 1994; 32(11): 2729-2737.

22. Bonnet R, Sampaio J L M, Labia R, De Champs C, Sirot D, Chanal C y Sirot J. A novel CTX-M $\beta$-Lactamase (CTX-M-8) in CefotaximeResistant Enterobacteriaceae Isolated in Brazil. Antimicrob Agents Chemother, 2000; 44(7): 1936-1942.
23. Paterson DL, Hujer K, Hujer A, Yeiser B, Bonomo M, Rice L, Bonomo R y The International Klebsiella Study Group. Extended-Spectrum $\beta$-Lactamases in Klebsiella pneumoniae Bloodstream Isolates from Seven Countries: Dominance and Widespread Prevalence of SHV- and CTX-M Type $\beta$ - Lactamases. Anticrob Agents Chemoter, 2003; 47(11): 3554-3560.

24. Casellas J. Resistencia a los antibacterianos en América Latina: consecuencias para la Infectología. Rev Panam Salud Pública, 2011; 30(6): 519 - 528.

25. Bush K, Jacoby G y Medeiros A. A Functional Classification Scheme for $\beta$-Lactamases and Its Correlation with Molecular Structure. Antimicrob Agents Chemother 1995; 39(6): 1211-1233.

26. Ambler RP. The Structure of $\beta$-Lactamases. Phil. Trans. R. Soc. Lond 1980; 289: 321-331.

27. Queenan A y Bush K. Carbapenemases: the Versatile $\beta$-Lactamases. Clin Microbiol Rev, 2007; 20(3): 440-458. 